\title{
Total ionizing dose effects on a highly integrated RF transceiver for small satellite radio applications in low earth orbit
}

\author{
Jan Budroweit, Maciej Sznajder* \\ German Aerospace Center (DLR), Institute of Space Systems, Avionic Systems, 28359 Bremen, Germany \\ *German Aerospace Center (DLR), Institute of Space Systems, Mechanics and Thermal Systems, 28359 Bremen, Germany \\ Phone: +49 42124420-1297, *+49 42124420-1623 Email: jan.budroweit@dlr.de, *maciej.sznajder@dlr.de
}

\begin{abstract}
Software-Defined Radios (SDR) are commonly used in state-of-the-art radio systems and are already well established in the space industry. A SDR usually describes the signal processing of a radio application in software and is often implemented into Field Programmable Gate Arrays (FPGA) or Digital Signal Processors (DSP). Most RF front-ends are strictly specified and realized for an executed application and are thus not re-configurable. With the release of new Radio Frequency Integrated Circuit (RFIC) devices, a significant portion of RF front end specifications have become programmable and alterable. This paper presents the use case and selected radiation test results of such RFIC technologies for small satellite radio applications, primary designed for low earth orbit (LEO) missions.
\end{abstract}

Keywords - SDR, Low Earth Orbit, RFIC, TID.

\section{INTRODUCTION}

Software-Defined Radios (SDR) have become an irreplaceable technology, even for the conservative and slow-developing space industry. Mainly, the RF front-end is the most limiting part in a radio system, since they are designed to dedicated requirements and they are often not re-configurable. A proposed solution to provide more flexibility is the multiplexing of different front-end modules, which increases the demands of power and size [1][2]. New Integrated Radio Frequency Integrated Circuit (RFIC) devices combine a wide range of RF front-end functionalities and are adjustable by software. Such benefits enable enormous flexibility for radio applications and re-define the definition of SDRs. Unfortunately, these devices are so far not designed for the harsh space environment. Thus, studies have to be made to analyze their susceptibility and reliability in space, in particular under radiation conditions [3]. In this paper, we present the Total Ionizing Dose (TID) test results for the AD9361, as a technical demonstrator for the new RFICs, used for small satellite radio applications in Low Earth Orbit (LEO) missions.

\section{TID LEVEL CALCULATION FOR LEO SMALL SATELLITE MISSIONS}

Estimation of the Total Ionization Dose (TID) level has been performed by use of the SPENVIS system [4]. First, both electron and proton fluxes have been calculated with an assumption of the following mission parameters. There were chosen a range of altitude from 300 to $800 \mathrm{~km}$, with an inclination angle of 98 degree. In all cases, mission time was chosen to last 1 year.

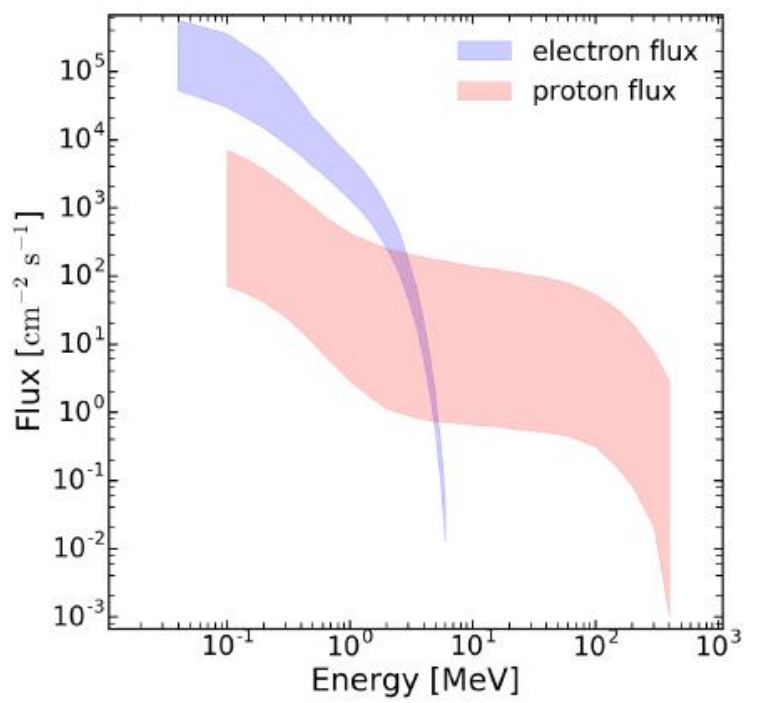

Fig. 1 Electron and proton fluxes from $300 \mathrm{~km}$ to $800 \mathrm{~km}$ altitude

The electron and proton fluxes are calculated by use of the AE-8 and AP-8 model respectively. Results are shown in the Fig. 1.

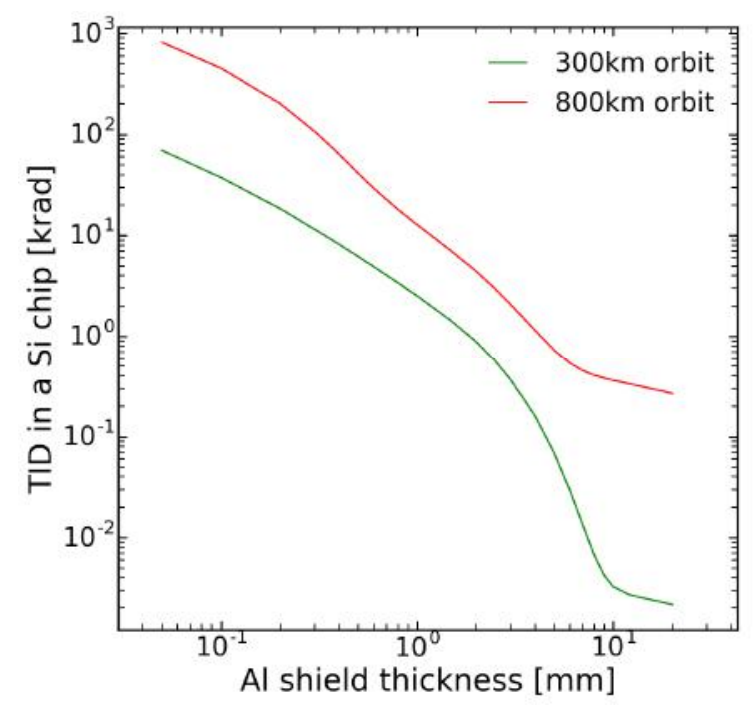

Fig. 2 TID as a function of Al shield thickness 
There, the lower boundary of the area corresponds to electron/proton flux at the $300 \mathrm{~km}$ orbit while the upper one to the $800 \mathrm{~km}$ orbit.

The particle fluxes are then used to calculate the TID by use of the SHIELDOSE-2 model [4]. It is calculated by assuming that a whiteness $\mathrm{Si}$-chip is placed in the center of an Al-sphere wall of $\mathrm{x}-\mathrm{mm}$ thick. The TID as a function of the shield thickness is shown in Fig. 2 for both orbits accordingly.

For $1 \mathrm{~mm}$ thick Al shield an arbitrary Si-chip will absorb approx. $12.5 \mathrm{kRad}$ per year at $800 \mathrm{~km}$ altitude. Therefore, for a typical LEO mission which lasts approx. two years the TID is $25 \mathrm{kRad}$.

\section{TID TEST ON THE AD9361 RFIC}

In this section we introduce the Device Under Test (DUT), an Agile Transceiver RFIC of Analog Devices. Moreover, the test setup for the TID test on the AD9361 is described and selected TID test results are presented and discussed.

\section{A. The AD9361 RFIC}

The device being tested is the AD9361 RF agile transceiver by Analog Devices [5]. The AD9361 is a 144-BGA plastic encapsulated IC with a dimension of $10 \times 10 \times 1.7 \mathrm{~mm}^{3}$. The DUT (AD9361BBCZ-ND, \#1350, 2769606.1, Singapore) is fabricated on a 7 -metal layer in a $65 \mathrm{~nm}$ CMOS process. A functional block design is presented in Fig. 3.

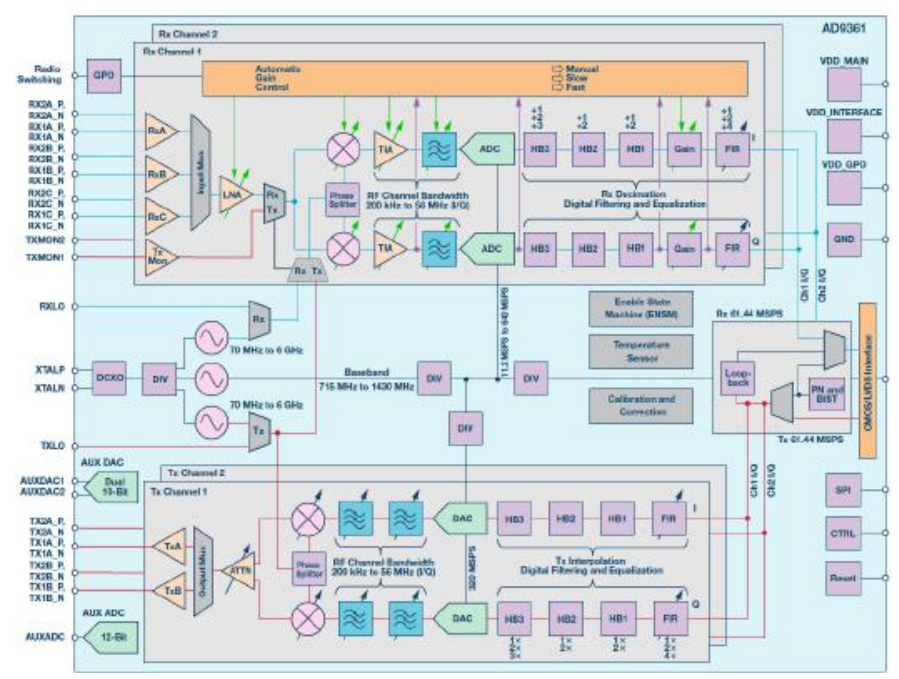

Fig. 3 Functional block design of the AD9361 DUT [6]

The RFIC consist of two independent transmitter and receiver chains, which are sharing a common clock source. This reference clock drives the analog digital converter (ADC) and the digital analog converter (DAC) circuits and the local oscillator (LO) control to mix the baseband signal into RF band. The LO frequency control $(70 \mathrm{MHz}$ to $6 \mathrm{GHz})$ is independent between the receiver and transmitter, whereby the selected sample rate (max. 128/64MSPS) is the same for both, receiver and transmitter. For each chain a stack of amplifiers and filters allows a narrow band selection and signal processing. The RFIC can be operated in Time Division Duplex (TDD) and Frequency Division Duplex (FDD) mode. In TDD mode the RFIC can receive or transmit data (half duplex), whereby in FDD mode a full duplex configuration is enabled. Each receiver and transmitter chain consists of selectable (by multiplexing) RF input (three) and RF outputs (two). All configurations of the AD9361 are programmable via SPI register control.

\section{B. TID test setup}

Based on the calculation of TID levels in section II, the selected test setup and parameters were chosen. The test facility uses a cobald-60 source to produce $\gamma$-rays irradiation. The Cobalt- 60 decays to nickel- 60 by emitting $\beta$ - and $\gamma$-rays. In this setup, the $\beta$-rays are absorbed by a steal shielding. The $\gamma$-rays have an energy level of $1.17 \mathrm{MeV}$ and $1.33 \mathrm{MeV}$.

There are four processes of interaction of electromagnetic radiation with matter: photoelectric effect, Compton Effect, generation of electron - positron pairs and photodisintegration or nuclear photoeffect. The gammas are responsible for the pair creation. Particle and its anti-particle are created while a photon passes the target nucleus in its close vicinity. This requirement is mandatory in order to conserve momentum and energy of the process. While the pair is created within a test object, the anti-particle annihilates with the regular matter and creates a quantum of electromagnetic radiation. The negatively charged particle of the pair contributes to the current initially applied to the DUT. This can obviously cause malfunctionality or even dilapidation of the DUT.

The test setup with the DUT is presented in Fig. 4. The AD9361 was placed on a test board (blue) and is attached to a baseband processor card (green). This card, and all non-to-irradiated devices, was shielded by a lead brick wall of $50 \mathrm{~mm}$ thickness. The distance to the cobald- 60 source was set to $50 \mathrm{~cm}$ to achieve a total dose of $25 \mathrm{kRad}$ within $24 \mathrm{~h}$ of continuous irradiation. The temperature during radiation exposure was $21^{\circ} \mathrm{C}\left(+/-1^{\circ}\right)$ all the time.
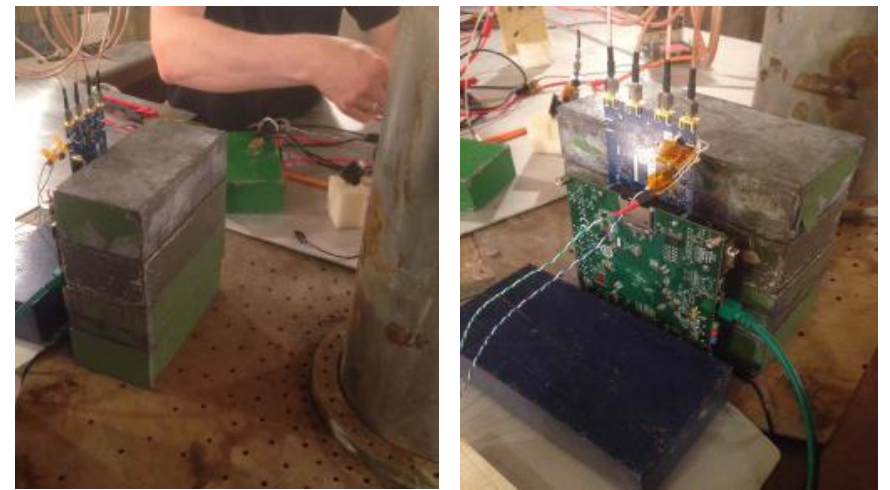

Fig. 4 TID test setup

The power to the DUT is provided by the baseband processing card. The AD9361 requires a supplied voltage of 1.3VDC. Important to notice is that the tolerance for this voltage is lower 
than $5 \%$. Thus, a very stable power supply is mandatory to avoid damages on the DUT. The test board with the DUT has two 1.3VDC voltage rails which are powering different function on 17 pins of the chip (e.g. RX synthesizer or TX LO). During irradiation the RFIC is set to a fix configuration (LO frequency, sample rate etc.) and the main objective is to monitor deviations on the analog and RF signal constraints. A schematic of the test setup is given in Fig. 5.

The baseband processing card is powered by a power supply on 12VDC. DUT related voltages and currents are observed with an analog signal module (NI9205) on a sample rate of $10 \mathrm{ksps}$. Voltages are monitored in single-ended configuration, whereby currents are observed in differential mode via voltage drop-off on shunt resistors $(\sim 30 \mathrm{~m} \Omega)$. During irradiation the receiver and transmitter paths of the DUT are cross connected to operate the device in FDD mode. All devices in the setup provide an Ethernet connection for control and data access. The test data are stored on a computer, located in the control room. In order to test and verify the performance of the DUT, the irradiation was paused at total doses of 3.5, 6, 11 and $25 \mathrm{kRad}$ to allow detailed performance measurements. For this purpose the test board with the DUT is demounted from the baseband processing board inside of the radiation chamber and is then mounted to another baseband processing board inside of test performance test bench (refer Fig. 5 in orange).

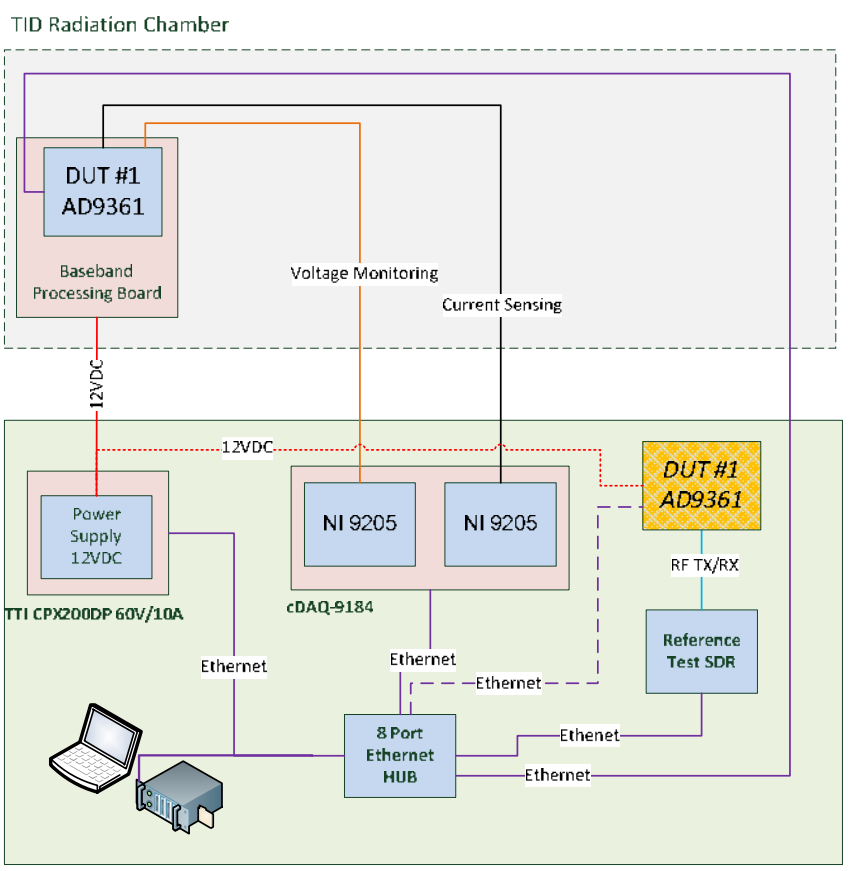

Control Room

Fig. 5 Schematic of the TID test setup

Part of the detailed performance test is the monitoring of the current and voltage signals for different operation modes (TDD, FDD, sleep etc.) and the analysis of receiver and transmitter related characteristic and properties. Due to the fact that the RFIC is capable to operate at a frequency range of $70 \mathrm{MHz}$ to $6 \mathrm{GHz}$, those performance tests were executed at three different LO frequencies: $800 \mathrm{MHz}, 2.4 \mathrm{GHz}$ and $5.5 \mathrm{GHz}$. A full frequency range test, or a better resolution with more LO frequencies, was not feasible due to the limited test time.

\section{TID test results}

During irradiation the AD9361 was operated in full FDD mode with both receivers and transmitters active to maximize the power consumption and system utilization. The analog signals of the AD9361, e.g. voltage and current levels, as well as the overall functional performance were monitored non-stop during the irradiation. Detailed performance test were executed on different radiation exposure levels.

\section{1) Analog signal}

To observe abnormalities in the DUT during irradiation, the supplied power (consisting of two 1.3VDC voltage rails) to the AD9361 were monitored. The voltage input level tolerance of the DUT is limited to $+/-30 \mathrm{mV}$. The conducted current on these voltage rails depends on the operational mode and may vary from $1 \mathrm{~mA}$ to $1 \mathrm{~A}$. Thus, during irradiation, only the full FDD mode was tested, whereas other modes were analyzed during the pauses. The voltage and current levels for the full FDD mode are presented in Fig. 6.
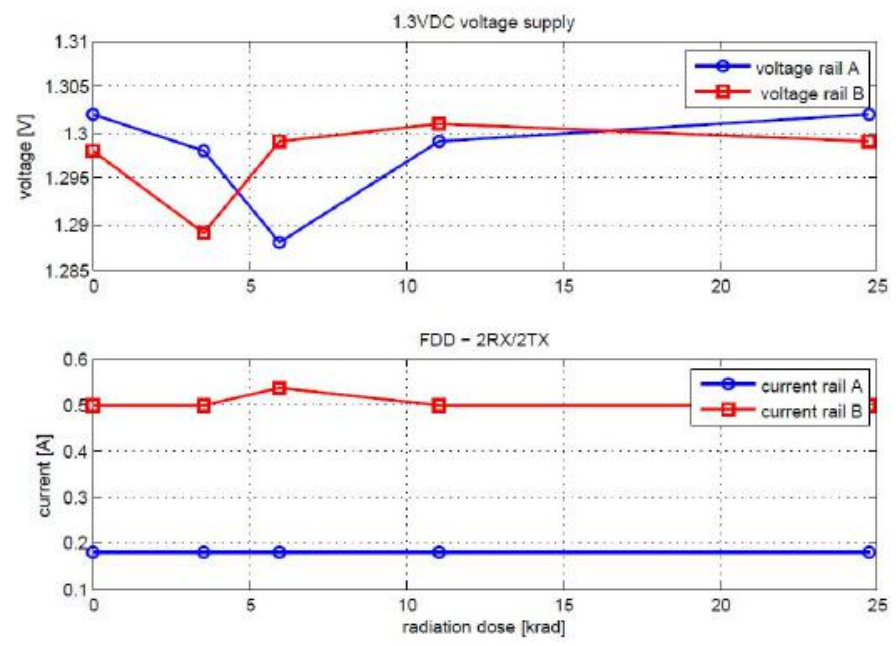

Fig. 6 Supplied voltages and current consumption vs. TID

The supplied voltage and current levels were stable during the complete irradiation test, up to the final dose of $25 \mathrm{kRad}$. This behaviour was also monitored for different operation modes at detailed performance test.

\section{2) Receiver performance}

To validate the receiver performance of the DUT, several performance tests were executed. In Fig. 7-9 the receivers manual gain control $(0 \mathrm{~dB}$ to $80 \mathrm{~dB})$ characteristic at different radiation doses are presented. The tests were performed at $800 \mathrm{MHz}, 2.4 \mathrm{GHz}$ and $5.5 \mathrm{GHz}$. As can be seen in Fig. 7, the gain function stays linear during a constantly transmitted signal ( $1 \mathrm{MHz}$ sinewave) to the receiver, at $800 \mathrm{MHz}$ carrier frequency. 

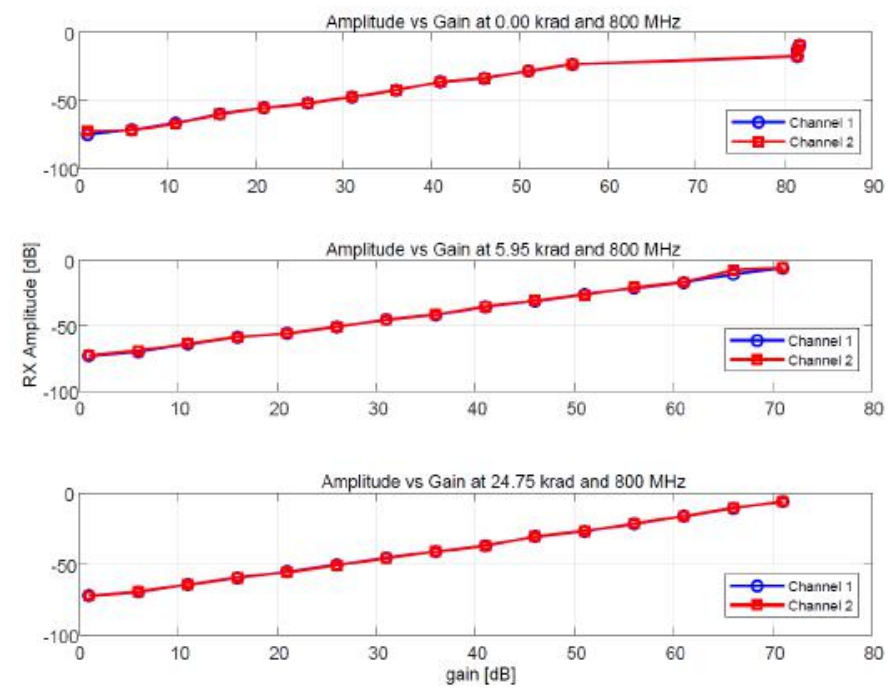

Fig. 7 RX amplitude - Gain function at $800 \mathrm{MHz}$ vs. TID.

Both channels of the DUT have been evaluated. In Fig. 8 the results for $2.4 \mathrm{GHz}$ carrier frequency are presented and Fig. 9 the test results for a $5.5 \mathrm{GHz}$.
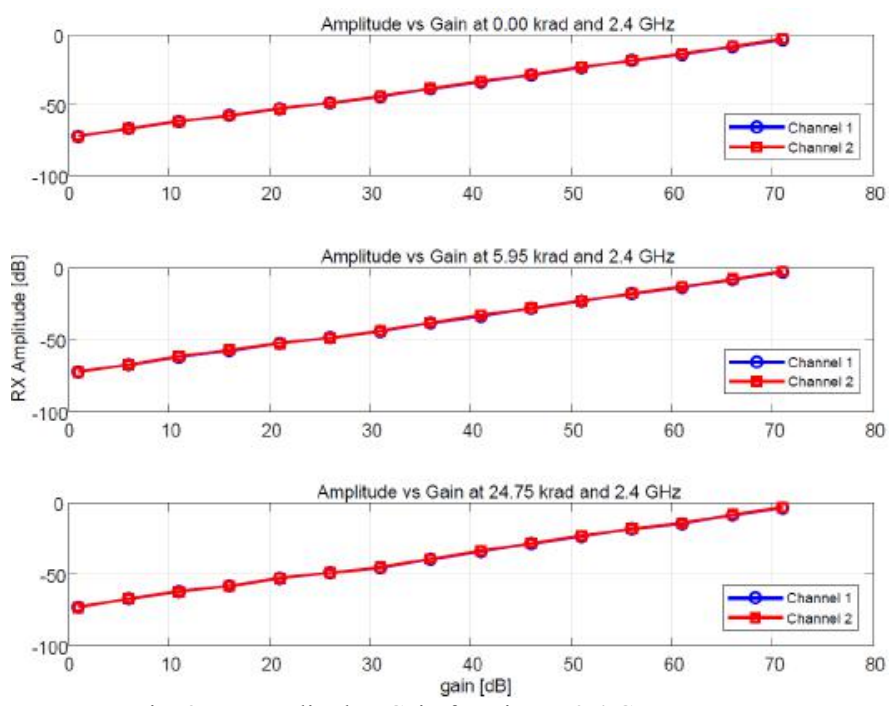

Fig. $8 \mathrm{RX}$ amplitude - Gain function at $2.4 \mathrm{GHz}$ vs. TID.

No degradation effect on the receiver's amplifier stages is detected for all three test frequencies.

Moreover, the SNR was measured during the gain control test to verify if the noise figure of the receiver input is affected by the radiation dose. For all test frequencies, the SNR was stable, as already observed in the gain functions of Fig. 7-9. Thus, the receiver's noise figure is also defined as not sensitive to TID of $25 \mathrm{kRad}$.

\section{3) Transmitter performance}

For the transmitter performance, different tests were executed to verify its functionality under irradiation. Fig. 10-12 show the TX Intermodulation Distortion (IMD) of a $1 \mathrm{MHz}$ and a $2 \mathrm{MHz}$ transmitted at different attenuation levels over the radiation dose.
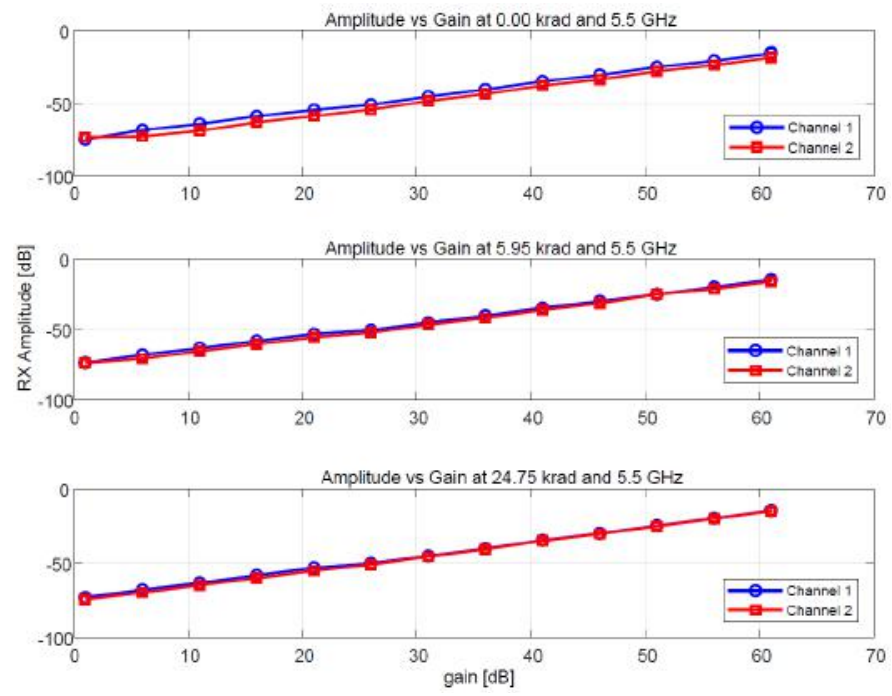

Fig. 9 RX amplitude - Gain function at $5.5 \mathrm{GHz}$ vs. TID.

IMD is the amplitude modulation of signal with two or more frequencies in a non-linear system. In this case the non-linearity is caused by the overdriven amplifier stage in the transmitter chain of the DUT. In Fig. 10 the IMD of the $3^{\text {rd }}$ and $5^{\text {th }}$ order at $-1 \mathrm{~dB},-11 \mathrm{~dB}$ and $-21 \mathrm{~dB}$ transmitter attenuation on a carrier frequency of $800 \mathrm{MHz}$ is presented
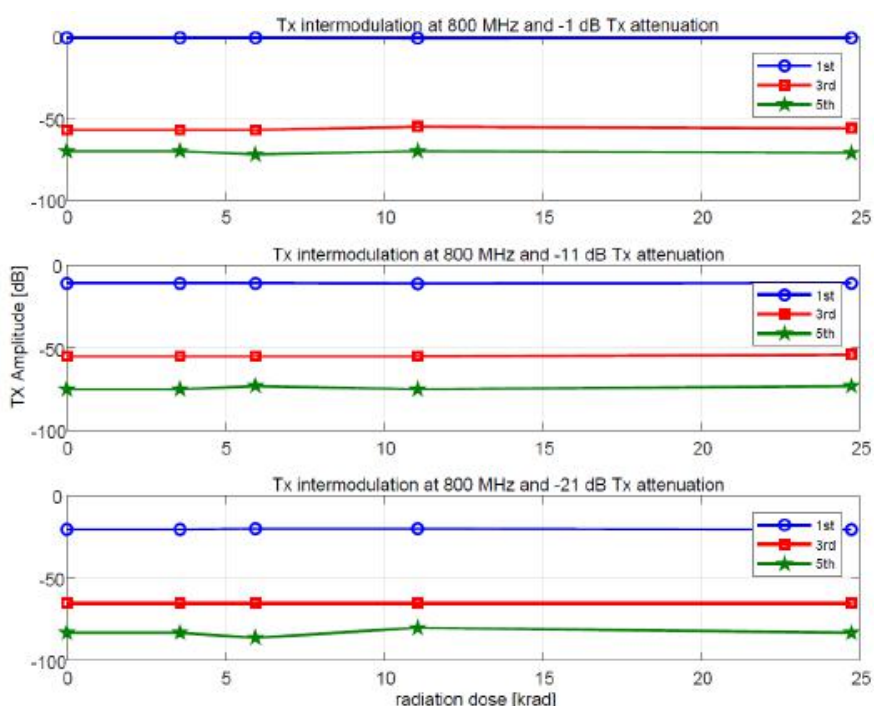

Fig. $10 \mathrm{TX}$ intermodulation test results at $800 \mathrm{MHz}$ vs. TID

At $-1 \mathrm{~dB}$ attenuation (near max. output power) the IMD distance for the fundamental tone and the $3^{\text {rd }}$ order is around $50 \mathrm{~dB}$. The $5^{\text {th }}$ order distance is approximately $75 \mathrm{~dB}$. These values stay constant over the full radiation exposure. The distance reduces with the decrease transmitter output power, controlled by the attenuation of the power amplifier.

At $2.4 \mathrm{GHz}$ frequency the results are similar as can be seen in Fig. 11. The intermodulation distances are frequencies depending. Thus, small differences between test results for different carrier frequencies are expected. 

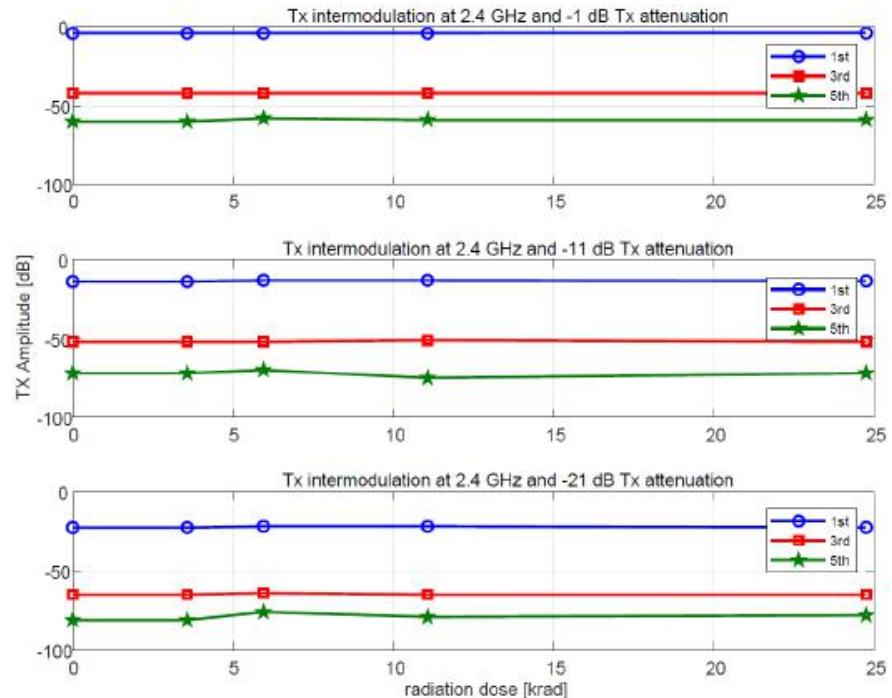

Fig. $11 \mathrm{TX}$ intermodulation test results at $2.4 \mathrm{GHz}$ vs. TID

For $2.4 \mathrm{GHz}$ the $3^{\text {rd }}$ distance is lower than $50 \mathrm{~dB}$ on an attenuation level of $-1 \mathrm{~dB}$. The test results for $5.5 \mathrm{GHz}$ carrier frequency is presented in Fig. 12. For all selected carrier frequencies, no degradation over the full radiated dose has been monitored. Another transmitter related test is the monitoring of the maximum transmitted power of a $1 \mathrm{MHz}$ signal tone on three different carrier frequencies.
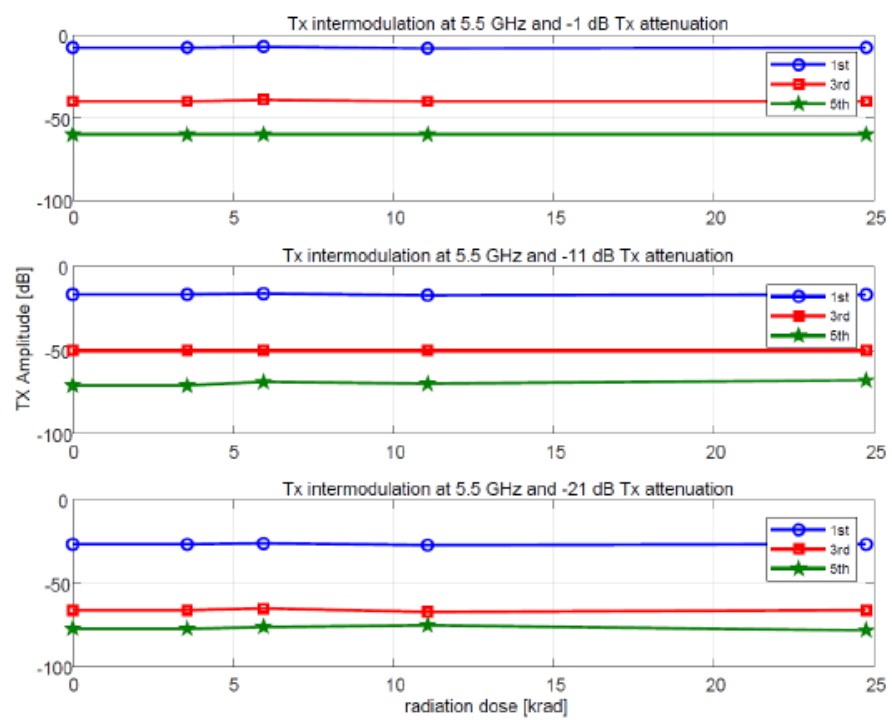

Fig. $12 \mathrm{TX}$ intermodulation test results at $5.5 \mathrm{GHz}$ vs. TID

In Fig. 13-15 the test results are presented for both transmitter outputs of the DUT. The output power is measured by a signal analyser configured on an average trace. Moreover, the frequency span was set to $10 \mathrm{MHz}$ to monitor the harmonic distortion. In Fig. 13 the output power on a carrier frequency of $800 \mathrm{MHz}$ is presented. As can be seen, there is a light deviation of the measured output power and a trend of continuous power drop with increasing radiation dose.

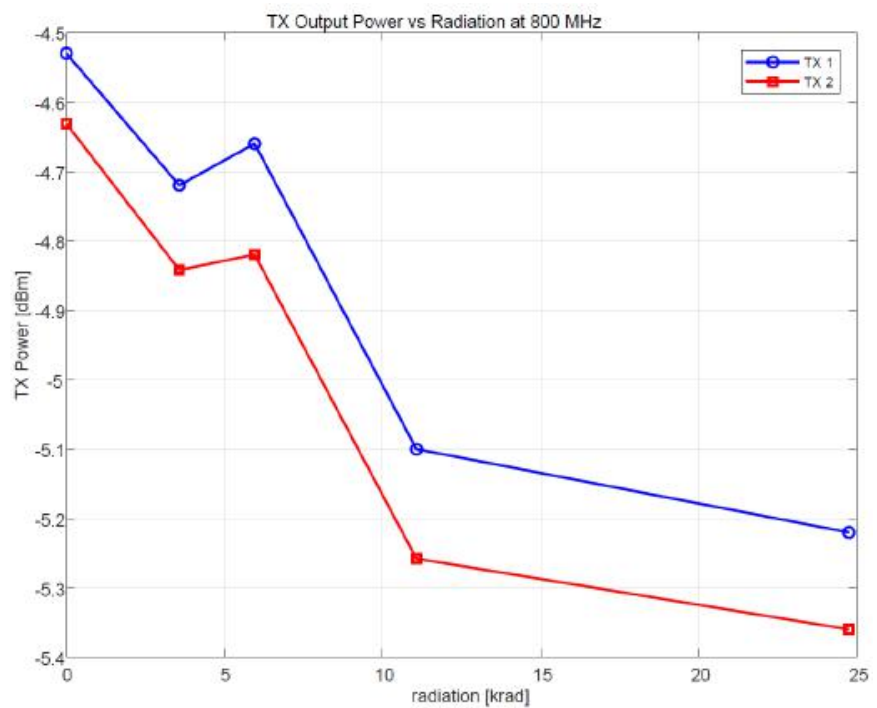

Fig. 13 Maximum output power at $800 \mathrm{MHz}$ vs. TID

The deviation can be manifested by the tolerances in the measurement technique $( \pm 1 \mathrm{~dB})$. Similar results are also presented for $2.4 \mathrm{GHz}$ in Fig. 14 and for $5.5 \mathrm{GHz}$ in Fig. 15. Also shown in here is the light deviation caused by the inaccuracy of measurement and a continuous drop of output power with increased radiation dose.

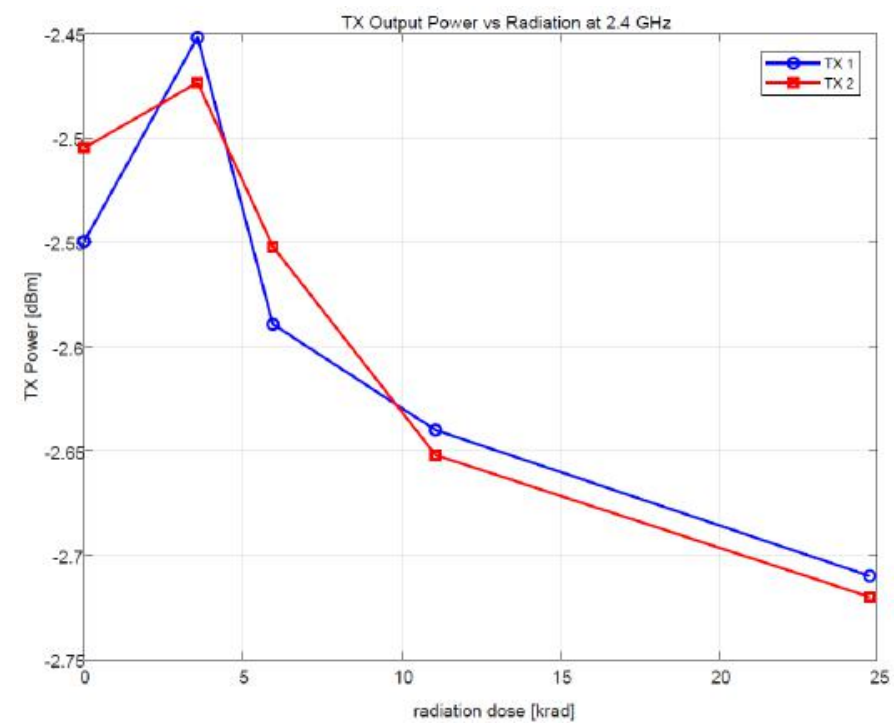

Fig. 14 Maximum output power at $2.4 \mathrm{GHz}$ vs. TID

Unfortunately, this effect could not be verified due to the limited radiation exposure to the DUT.

All in all, no degradation effects were observed, neither on the attenuation-block, nor the up-converter stage of the two transmitter chains. 


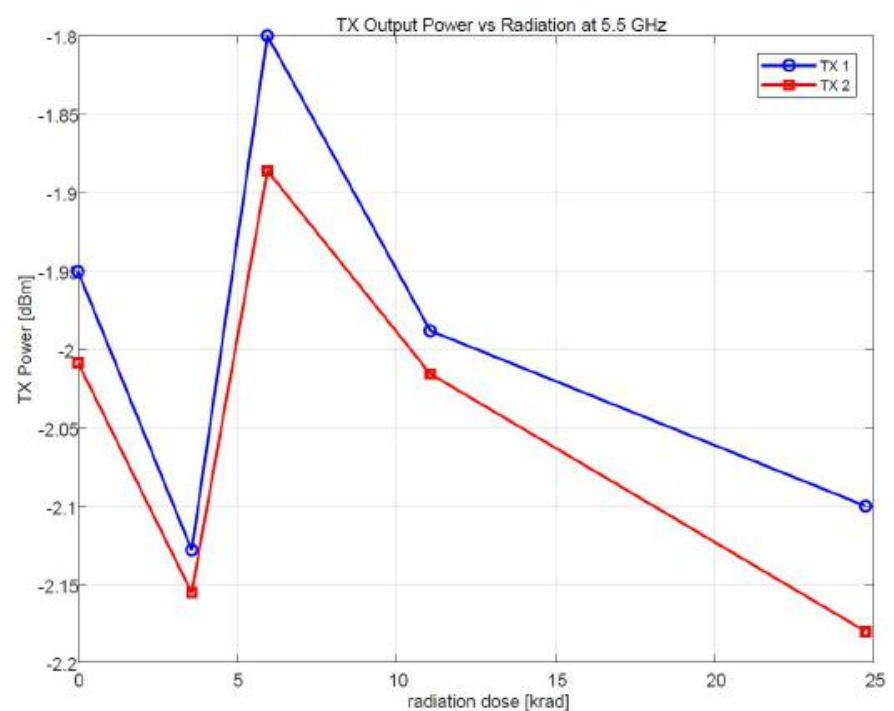

Fig. 15 Maximum output power at $5.5 \mathrm{GHz}$ vs. TID

\section{CONCLUSION}

In this paper we presented the use of new RFICs for small satellite radio applications and TID test results. The results show that no conspicuous degradation effects on the DUT were observed up to $25 \mathrm{kRad}$ total ionizing dose. Upcoming activities are the evaluation on TID effects for higher dose levels (deep-space missions) and Single Event Effects (SEE) characterization.

\section{REFERENCES}

[1] J. Budroweit, "Software-defined radio with flexible RF front end for satellite maritime radio applications," published in CEAS Space Journal (2016) 8: 201. doi:10.1007/s12567-016-0121-9.

[2] J. Budroweit, "Design of a Highly Integrated and Reliable SDR Platform for Multiple RF Applications on Spacecrafts," GLOBECOM 2017 - 2017 IEEE Global Communications Conference, Singapore, 2017, pp. 1-6. doi: 10.1109/GLOCOM.2017.8255087

[3] J. Budroweit and A. Koelpin, "Design challenges of a highly integrated SDR platform for multiband spacecraft applications in radiation environments," 2018 IEEE Topical Workshop on Internet of Space (TWIOS), Anaheim, CA, 2018, pp. 9-12. doi: 10.1109/TWIOS.2018.8311399

[4] SPENVIS, www.spenvis.oma.be .

[5] Analog Devices, Software-Defined Radio Solutions from Analog Devices, 2014

[6] D. Pu, A. Cozma and T. Hill, "Four Quick Steps to Production: Using Model-Based Design for Software-Defined Radio (Part 1), " Analog Dialogue 49-09, September 2015 